\title{
Acidity Constants and its Dependence on Solvent Selection from First-principles Calculations using Cluster-Continuum Models
}

\author{
Hieu H. Pham*, Christopher D. Taylor, Neil J. Henson \\ Los Alamos National Laboratory, Los Alamos, New Mexico, USA 87544
}

\begin{abstract}
Dissociation constants of selected carboxylic acids in aqueous and organic solvents were calculated at quantum chemical level. We considered cases in which trace quantities of water may be present, as well as cases in which water was entirely absent. In the latter cases, alternative proton acceptors need to be considered. For aqueous solvent, short-range solvation effects are considered by adding explicit water molecules as the first solvent shell. In the absence of water, corresponding organic solvents are used directly as the proton acceptors and the resulted $\mathrm{pK}_{\mathrm{a}}$ are quite comparable to those obtained from previous case of aqueous solvent.
\end{abstract}

Key words: pKa, dissociation constant, carboxylic acids, solvent effect, dielectric constant

*Corresponding author:

E-mail: hieuhpham@gmail.com

Tel: $510-486-5178$ 


\section{Introduction}

The strength of an acid in solution is quantified by its tendency to dissociate into constituent ions, which is in general measured by the logarithm of the acid dissociation constant, $\mathrm{pK}_{\mathrm{a}}$. This is usually expressed relative to the parent acid's behavior in aqueous solution, but the question emerges as to its capability to be applied to other solvents. The acidity of chemical compounds is a prerequisite in understanding many fundamental phenomena in chemistry, biological science and medicine, and the knowledge of this thermodynamic property finds applications in control of many industrial processes, including chemical and pharmaceutical manufactures $[1,2]$. The dissociation constants of carboxylic acids in aqueous solution can be experimentally obtained by measurements of conductance $[3,4]$, calorimetry [5], potentiometry [6-9] and spectroscopy [10]. In some cases, the experimental methods can be restricted, for instance, to a certain range of temperatures, $\mathrm{pH}$ and $\mathrm{pK}_{\mathrm{a}}$. These measurements are also complicated when substance's solubility in solution is low or when the surrounding environment is complex $[1,11,12]$.

The $\mathrm{pK}_{\mathrm{a}}$ of a given acid can be usefully calculated using a quantum chemical method. Many studies on dissociation constants of organic compound in solution have been recently reported [1,13-21]. Computational techniques and methods have been recently reviewed and it was shown that the accuracy of obtained $\mathrm{pK}_{\mathrm{a}}$ values could be highly subject to the choice of thermodynamic cycles and solvation models, including direct methods, proton exchange scheme, cluster-continuum model and implicit-explicit models [22-24]. The equilibrium of acid dissociation into a proton and the associated conjugated base can be simply described in the following equation:

$H A \rightarrow H^{+}+A^{-}$

The equilibrium constant of the acid dissociation process, $\mathrm{K}_{\mathrm{a}}$, is defined as:

$K_{a}=\frac{\left[H^{+}\right]\left[A^{-}\right]}{[H A]}$

The $\mathrm{pK}_{\mathrm{a}}$ is then obtained by

$p K_{a}=-\log _{10} K_{a}$

, where HA denotes the parent acid, $\mathrm{A}^{-}$is the conjugate base and $\mathrm{H}^{+}$is the proton. Several authors have directly employed this thermodynamic cycle in their theoretical investigations $[11,15,16]$. The limitation of this approach is that solvation energy of a free proton is not a calculable quantity quantum chemically, since it has no electrons. The 
commonly used workaround is to use the experimentally derived absolute solvation free energy of the proton. Although this process has been shown to work well for acids in aqueous solution, it is clearly inappropriate for other solvents since the solvation free energy will not commonly be available. A more often and recently used approach suggests to recast the dissociation process to involve an explicit water molecule. The proton is now represented by the hydronium ion, $\mathrm{H}_{3} \mathrm{O}^{+}$:

$\mathrm{HA}+\mathrm{H}_{2} \mathrm{O} \rightarrow \mathrm{H}_{3} \mathrm{O}^{+}+\mathrm{A}^{-}$

, the appropriate equilibrium constant is:

$K^{\prime}=\frac{\left[\mathrm{H}_{3} \mathrm{O}^{+}\right]\left[\mathrm{A}^{-}\right]}{[\mathrm{HA}]\left[\mathrm{H}_{2} \mathrm{O}\right]}$

, where we can relate this to $\mathrm{K}_{\mathrm{a}}$ by

$K^{\prime}=\frac{K_{a}}{\left[\mathrm{H}_{2} \mathrm{O}\right]}$

Clearly this model may work when water is the proton acceptor species, and, in many non-aqueous solvents, there is likely to exist some trace amount of water, at the part-per-million (ppm) level, for instance. In such cases, equation (6) could be usefully applied. In fact, numerous first-principles studies of deprotonation of organic and biochemical compounds in nonaqueous solutions were already presented [25-28]. The authors either used the direct thermodynamic cycle, as presented in equation (1), or used the super-molecule approach, in which a well-known compound is used as the reference for the pka estimation of the desired compound. The continuum model was then incorporated to account for the solvation effect from nonaqueous solvents implicitly. In this work, however, we consider the capability for other species (nonaqueous solvents) to accept proton, in the absence of water. Although a diversity of possible proton-accepting contaminants may exist, we herein constrain ourselves to consider the possibility for proton complexation by a variety of neat solvent molecules, including heptane, carbontetrachloride, benzene, chloroform, dichloroethane and acetone. This investigation spans a range of alkane, aromatic and halogenated solvents at the low end of dielectric constants (below 20). In such study we consider two possibilities. In the first case, the hydrogen cation binds to some number $n$ solvent molecules. When we investigated these cases, we found that the proton in fact chemically adds to hydrocarbons to form carbocation: 
$R C O O H+n R^{\prime} \rightarrow H^{+}\left[R^{\prime}\right]_{n}+R \mathrm{COO}^{-}$

$K_{a, \text { solv }}=\frac{\left[\mathrm{H}^{+}\right]\left[\mathrm{RCOO}^{-}\right]}{[\mathrm{RCOOH}]}$

In other cases, the $\mathrm{H}+$ can bind to a basic functional group (B) in the solvent molecule, resulting in the elimination of $\mathrm{BH}$ and the formation of a carbocation via the reaction:

$\mathrm{RCOOH}+\mathrm{B} \cdots \mathrm{R}^{\prime} \rightarrow \mathrm{BH}+\mathrm{R}^{+}+\mathrm{RCOO}^{-}$(9)

$K_{a, s o l v}=\frac{[B H]\left[R^{\prime+}\right]\left[\mathrm{RCOO}^{-}\right]}{[\mathrm{RCOOH}]}$

One of the less computationally expensive methods to account for the effect of the surrounding solvent medium, beyond such reaction centers, is by using the continuum model. This method is widely used by computational community due to its simplicity and broad physical meaning that allows the treatment of basic chemical properties in liquid phase, especially for neutral species [29]. However, the homogeneous continuum, while introduces an average effect induced by solvent medium (representing by internal properties such as dielectric constant and thermal expansion), does not capture all the details of the individual bonding interactions between solute and solvent molecules. This is even more challenging when dealing with ionic species due to its strong electrostatic interaction with the environment. As an attempt to overcome this drawback, several authors recently showed that the $\mathrm{pK}_{\mathrm{a}}$ calculation could be improved by using a so-called cluster-continuum model $[12,14,18,19]$. In this extended method, the use of explicit solvent molecules is combined with surrounding continuum to account for the bulk solvent effects on dissociation products.

Some success has been obtained for $\mathrm{pK}_{\mathrm{a}}$ calculations at normal conditions, utilizing all the techniques mentioned above. However, many industrial processes are carried out in environments other than aqueous. In this work, we report our attempt on theoretical calculations of dissociation constants for carboxylic acids in both aqueous solution and organic solvents. The next section will detail the theoretical and computational background, followed by our results and discussions in section III. 

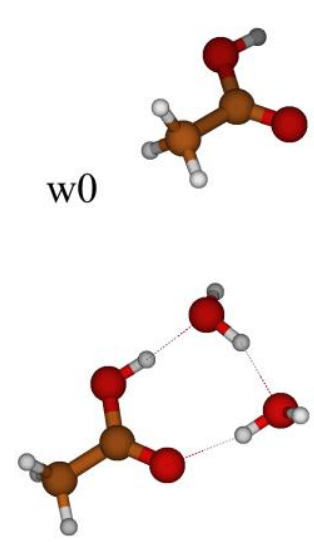

w11

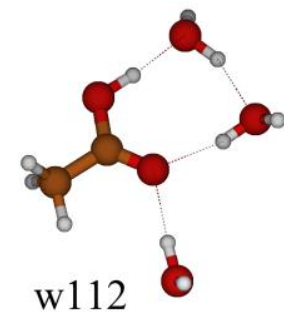

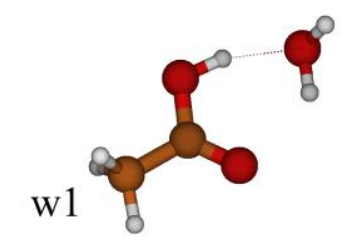

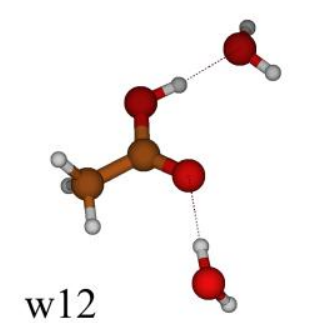

w12

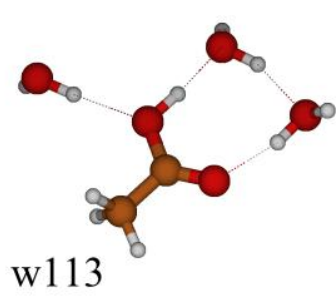

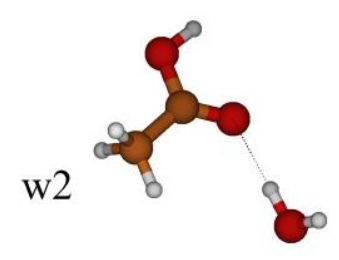
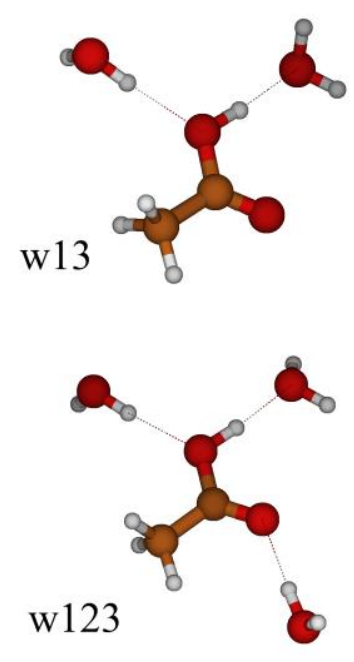
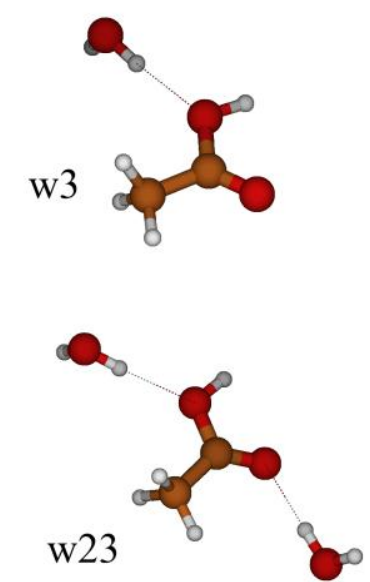

w23

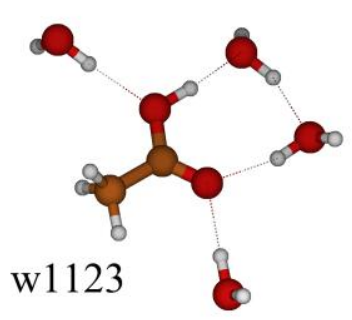

Figure 1. Optimized structures of hydrated acetic acid clusters in aqueous solution, performed using the B3LYP/PCM/6-31+G(d,p) method

\section{Theoretical and Computational Details}

First-principles quantum chemical calculations were performed using the Gaussian 09 software [30]. Geometry optimization and free energy are computed mainly at the DFT/B3LYP level of theory using a 6-31+G(d,p) basis set [31], otherwise noted. Implicit solvation effects were incorporated using the self-consistent reaction field (SCRF) method with a polarized continuum model (PCM) [29], in which the aqueous medium is modeled as a continuum of uniform dielectric constant $\varepsilon$. The solute supermolecules or solvation clusters are immersed in a cavity with appropriate shape embedded in an infinite polarizable dielectric medium. Besides, another continuum solvation model called SMD [32], which is based on self-consistent reaction field treatment of quantum mechanical charge density, was also used to perform calculations at M06-2X/6-311+G** electronic structure method for comparison purpose. 


$$
\begin{aligned}
& H A\left(H_{2} \mathrm{O}\right)_{n}(\text { gas })+H_{2} \mathrm{O}(\text { gas }) \stackrel{\Delta G_{\text {gas }}^{0}}{\longrightarrow} H_{3} \mathrm{O}^{+}(\text {gas })+A^{-}\left(\mathrm{H}_{2} \mathrm{O}\right)_{n}(\text { gas })
\end{aligned}
$$

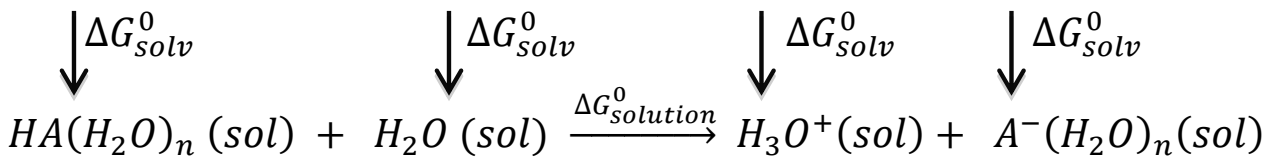

Figure 2. Thermodynamic cycle corresponding to the dissociation of a proton from acid cluster with explicit water molecules.

\section{Cluster model of explicit water incorporation}

The explicit interaction between carboxylic group and surrounding aqueous medium is accounted for, by coordinating water molecules at different positions (Figure 1). Three locations could be found for a single water molecule to coordinate with the acid group, as shown by Du et al. [14], therefore there are a total eleven possible acid-water clusters, in addition to the un-hydrated model (w0 in Figure 1). The corresponding models for conjugate base - water clusters will be similar, with the hydration number up to four.

\section{Thermodynamic cycle and calculations of dissociation constants}

The Gibbs free energy of the dissociation reaction in gas phase $\left(\Delta G_{g a s}^{0}\right)$ of a molecule (or cluster) is obtained by subtracting the total energy of reactants from that of dissociation products.

$\Delta \mathrm{G}_{\text {gas }}^{0}=\mathrm{G}_{\text {gas }}^{0}\left(\mathrm{H}_{3} \mathrm{O}^{+}\right)+\mathrm{G}_{\text {gas }}^{0}\left(\mathrm{~A}^{-}\left(\mathrm{H}_{2} \mathrm{O}\right)_{\mathrm{n}}\right)-\mathrm{G}_{\text {gas }}^{0}\left(\mathrm{H}_{2} \mathrm{O}\right)+\mathrm{G}_{\text {gas }}^{0}\left(\mathrm{HA}\left(\mathrm{H}_{2} \mathrm{O}\right)_{\mathrm{n}}\right)$

Using equation (4) with an explicit water molecule, the thermodynamic cycle of dissociation process for the acid cluster is represented in Figure $\mathbf{2}$ and the dissociation energy in solution can be obtained as follows:

$$
\begin{aligned}
& \Delta \mathrm{G}_{\text {solution }}^{0}=\Delta \mathrm{G}_{\text {gas }}^{0}+\Delta \Delta \mathrm{G}_{\text {solv }}^{0} \\
& =\Delta \mathrm{G}_{\text {gas }}^{0}+\Delta \mathrm{G}_{\text {solv }}^{0}\left(\mathrm{H}_{3} \mathrm{O}^{+}\right)+\Delta \mathrm{G}_{\text {solv }}^{0}\left(\mathrm{~A}^{-}\left(\mathrm{H}_{2} \mathrm{O}\right)_{\mathrm{n}}\right)-\Delta \mathrm{G}_{\text {solv }}^{0}\left(\mathrm{H}_{2} \mathrm{O}\right)-\Delta \mathrm{G}_{\text {solv }}^{0}\left(\mathrm{HA}\left(\mathrm{H}_{2} \mathrm{O}\right)_{\mathrm{n}}\right)
\end{aligned}
$$

The solvation free energies are obtained by performing calculations on a given species both in the gas phase and using the model for the dielectric environment produced by the surrounding solvent. For $\Delta G_{\text {solv }}^{0}\left(\mathrm{H}_{3} \mathrm{O}^{+}\right)$in aqueous solution, we used the experimental value of $-110.2 \mathrm{kcal} / \mathrm{mol}$, since several studies have concluded that this reproduces more 
accurate $\mathrm{pK}_{\mathrm{a}}$ values $[13,14]$. For other organic solvents, we just used the values obtained from quantum chemical calculations.

The $\mathrm{pK}_{\mathrm{a}}$ is then obtained directly from the Gibbs free energy, using the following relation:

$\Delta G_{\text {solution }}^{0}=-2.303 R T \log \frac{K_{a}}{\left[\mathrm{H}_{2} \mathrm{O}\right]}$

At room temperature, the reference state of water is taken as $\left[\mathrm{H}_{2} \mathrm{O}\right]=55.4 \mathrm{M}[13]$ and the relation becomes:

$p K_{a}=\frac{\Delta G_{\text {solution }}^{0}-2.38}{1.364}$

\section{Cluster model for non-aqueous proton acceptors}

For non-aqueous solvents, we suggest that it is important to also consider the possibility for an organic molecule to accept the proton from the carboxylic acid.

Although such events are expected to be significantly endothermic, this does not preclude small quantities of conjugate base being formed under equilibrium conditions. We therefore construct molecular models for the proton adjacent to the non-aqueous solvent species and performed geometry optimization in order to determine the preferred proton solvation configuration. In all cases it is found that the proton prefers to chemically interact with the organic molecule. In particular, we find two possible thermodynamic cycles emerge, as demonstrated in Figure 3.

$$
\begin{aligned}
& (I): \quad H A(\text { gas })+R(\text { gas }) \stackrel{\Delta G_{\text {gas }}^{0}}{\longrightarrow} H R^{+}(\text {gas })+A^{-}(\text {gas }) \\
& \downarrow \Delta G_{\text {solv }}^{0} \quad \downarrow \Delta G_{\text {solv }}^{0} \quad \downarrow^{\Delta G_{\text {solv }}^{0}} \quad \downarrow^{\Delta G_{\text {solv }}^{0}} \\
& H A(\mathrm{sol})+R(\mathrm{sol}) \stackrel{\Delta G_{\text {solution }}^{0}}{\longrightarrow} \mathrm{HR}^{+}(\mathrm{sol})+A^{-}(\mathrm{sol}) \\
& \text { (II): } \quad H A(\text { gas })+B \cdots R(\text { gas }) \stackrel{\Delta G_{g a s}^{0}}{\longrightarrow} H B(\text { gas })+R^{+}(\text {gas })+A^{-}(\text {gas }) \\
& \downarrow \Delta G_{\text {solv }}^{0} \quad \downarrow^{\Delta} G_{\text {solv }}^{0} \quad \downarrow^{\Delta G_{\text {solv }}^{0}} \quad \downarrow^{\Delta G_{\text {solv }}^{0}} \quad \downarrow^{\Delta G_{\text {solv }}^{0}} \\
& H A(\mathrm{sol})+B \cdots R(\mathrm{sol}) \stackrel{\Delta G_{\text {solution }}^{0}}{\longrightarrow} H B(\mathrm{sol})+R^{+}(\mathrm{sol})+A^{-}(\mathrm{sol})
\end{aligned}
$$

Figure 3. Thermodynamic cycles corresponding to the dissociation of a proton from acid cluster with non-aqueous proton acceptors 
The $p K_{a}$ is then obtained from the Gibbs free energy, using the relation:

$\Delta G_{\text {solution }}^{0}=-2.303 R T \log \frac{K_{a}}{[R]}$

, where reference $[R]$ (or $[B-R]$ in the cycle II) is approximated as the standard state of the corresponding proton-acceptor solvent.

\section{Results and Discussion}

\subsection{Model selection and water aggregates}

Stable structures of hydrate supermolecules are found in both gas phase and solvent phases (Figure 1). The clustering of water molecules at position 1 forms a ring structure. However, in strong solvent such as aqueous solution, further incorporation of water into positions 2 or 3 will disrupt the ring structure of monohydrate with water at position 1. As seen in configurations w12, w13 and w123, the water molecule at position 1 forms only one hydrogen bond with the carboxyl group.

The solvation free energies $\Delta \mathrm{G}_{\text {solv }}$ of parent water-acid clusters and their respective anions (in aqueous solution) are tabulated in Table 1, using both B3LYP/PCM/6-31+G(d,p) (hereafter shorten as PCM) and M06-2X/SMD/6-311+G** (hereafter shorten as SMD) methods. These $\Delta \mathrm{G}_{\text {solv }}$ are more negative for highly hydrated super-molecules. At the same hydration number, the increase in the absolute value of $\Delta \mathrm{G}_{\text {solv }}$ could be observed in the order: position $1<$ position $2<$ position 3 . In general, the increase in hydration number corresponds to the increase in absolute $\Delta \mathrm{G}_{\text {solv }}$ except the case of two waters at position 1. For instance, the solvation free energies of w112 and w113 structures are comparable to those of w12 and w13. The solvation energies calculated using M06-2X/SMD/6-311+G** are more negative than those calculated using B3LYP/PCM/6-31+G(d,p).

Due to the charge and high dipole of the conjugate base molecules, there are large interactions between the conjugate bases and solvents; therefore their solvation results in the release of high energy (Table 1). However, the effect of hydration was found to exibit the opposite trend, compared to the non-charged acids: highly hydrated anions correspond to less negative solvation free energies. It was proposed that the surrounding waters act as barriers to reduce the interaction between the anion and environment [14]. 
It was suggested by earlier theoretical studies that treating water molecules explicitly in the first solvation shell is required to account for strong solute-solvent interactions [18]. Table 2 summaries the $\mathrm{pK}_{\mathrm{a}}$ calculated for different cluster models and their error compared to experimental values. In general, calculations using non-hydrate models yield high $\mathrm{pK}_{\mathrm{a}}$ but the use of cluster models results in significant accuracy improvement. In general, highly hydrated clusters are more acidic and this indicates that hydrogen bonding with water molecules weakens the $\mathrm{O}-\mathrm{H}$ bond in the carbonyl group and induces stronger proton dissociation. The effect of water association on increasing the acidity can be seen in the order: position $1<$ position $2<$ position 3 , and so in $11<12$ $<13<23$ and $112<113$. When water is incorporated at position 3, it forms the hydrogen bond directly with $\mathrm{O}$ of the carbonyl group (see Figure 1), therefore draws charge to itself and subsequently induces a more pronounced effect to the ability to donate proton.

Table 1. Solvation free energies $\Delta \mathrm{G}_{\text {solv }}(\mathrm{kcal} / \mathrm{mol})$ of parent acids and respective conjugate bases in aqueous solution, calculated with PCM and SMD (given in parentheses)

\begin{tabular}{|l|l|l|l|l|}
\hline \multirow{2}{*}{$\begin{array}{l}\text { cluster } \\
\text { models }\end{array}$} & Acetic acid & \multicolumn{1}{l|}{ Benzoic acid } \\
\cline { 2 - 5 } parent acid & cjg. base & parent acid & cjg. base \\
\hline w1 & $-5.2(-7.8)$ & $-65.2(-72.1)$ & $-5.2(-7.3)$ & $-61.3(-67.2)$ \\
\hline w2 & $-6.6(-10.6)$ & $-58.8(-66.3)$ & $-6.7(-11.0)$ & $-56.3(-65.2)$ \\
\hline w3 & $-8.6(-12.4)$ & $-61.2(-69.6)$ & $-9.1(-12.6)$ & $-58.4(-65.3)$ \\
\hline w11 & $-9.6(-13.5)$ & $-61.2(-69.6)$ & $-9.9(-15.1)$ & $-58.4(-65.3)$ \\
\hline w12 & $-7.7(-12.5)$ & $-57.4(-65.7)$ & $-7.9(-13.1)$ & $-55.1(-62.6)$ \\
\hline w13 & $-11.6(-16.4)$ & $-57.1(-67.3)$ & $-11.9(-17.1)$ & $-55.3(-63.7)$ \\
\hline w23 & $-12.1(-17.1)$ & $-57.1(-67.3)$ & $-12.3(-17.8)$ & $-55.3(-63.7)$ \\
\hline w112 & $-11.8(-17.9)$ & $-56.6(-64.1)$ & $-13.7(-16.4)$ & $-54.8(-61.7)$ \\
\hline w113 & $-11.9(-18.5)$ & $-56.6(-64.1)$ & $-12.0(-19.2)$ & $-54.8(-61.7)$ \\
\hline w123 & $-16.3(-22.9)$ & $-56.3(-63.6)$ & $-16.4(-23.8)$ & $-54.2(-62.4)$ \\
\hline w1123 & $-15.8(-23.9)$ & $-54.9(-63.9)$ & $-17.4(-21.9)$ & $-55.3(-65.4)$ \\
\hline
\end{tabular}

It was shown in Table 2 that M06-2X/SMD/6-311+G** electronic structure method yields relatively large $\mathrm{pK}_{\mathrm{a}}$ values. Hydrate models with acceptable errors compared to experimental data are w23 (two water clustering) and w1123 (four water 
clustering). However, at B3LYP/PCM/6-31+G(d,p) level, a hydration number of three was optimal in reproducing experimental acidity data (w112, w113, w123) and this is consistent with the suggestion by Du et al. (w123), using the same functional [14]. In addition, the use of model w13 with two water molecules also reproduces a relatively accurate dissociation constant, with an error of less than one order of magnitude (highlighed in bold). In general, using the B3LYP/PCM/6-31+G(d,p) method, models with less than two water molecules correspond the $\mathrm{pK}_{\mathrm{a}}$ that are too high and clusters with a hydration number of four have quite low $\mathrm{pK}_{\mathrm{a}}$ values compared to experiments.

Table 2. The calculated pKa values of acetic and benzoic acids with PCM and SMD (given in parentheses). Errors less than one order of magnitude (compared to experimental data) are highlighted in bold.

\begin{tabular}{|c|c|c|c|c|}
\hline \multirow{2}{*}{$\begin{array}{l}\text { Cluster } \\
\text { models }\end{array}$} & \multicolumn{2}{|l|}{ Acetic acid } & \multicolumn{2}{|c|}{ Benzoic acid } \\
\hline & $p K_{a}$ & error & $p K_{a}$ & error \\
\hline w0 & $8.83(9.63)$ & $4.07(4.87)$ & $7.47(7.26)$ & $3.27(3.06)$ \\
\hline w1 & $7.47(8.69)$ & $2.71(3.93)$ & $6.16(7.27)$ & $1.96(3.07)$ \\
\hline w2 & $6.80(7.10)$ & $2.04(2.34)$ & $5.86(6.18)$ & $1.66(1.98)$ \\
\hline w3 & $6.10(6.71)$ & $1.34(1.95)$ & $5.09(6.47)$ & $0.89(2.01)$ \\
\hline w11 & $6.76(8.51)$ & $2.00(3.75)$ & $5.95(6.90)$ & $1.75(2.70)$ \\
\hline $\mathrm{w} 12$ & $6.14(7.74)$ & $1.38(2.98)$ & $5.24(6.92)$ & $1.04(2.72)$ \\
\hline w13 & $5.58(7.39)$ & $\mathbf{0 . 8 2}(2.63)$ & $4.64(6.48)$ & $0.44(2.28)$ \\
\hline $\mathrm{w} 23$ & $4.01(5.67)$ & $-0.75(\mathbf{0 . 9 1})$ & $2.43(4.53)$ & $-1.77(\mathbf{0 . 3 3})$ \\
\hline w112 & $4.72(7.29)$ & $\mathbf{- 0 . 0 4}(2.53)$ & $4.71(6.33)$ & $0.51(2.13)$ \\
\hline w113 & $4.24(7.17)$ & $\mathbf{- 0 . 5 2}(2.41)$ & $3.98(6.04)$ & $\mathbf{- 0 . 2 2}(1.84)$ \\
\hline w123 & $3.88(7.02)$ & $\mathbf{- 0 . 8 8}(2.26)$ & $3.61(5.22)$ & $\mathbf{- 0 . 5 9}(1.02)$ \\
\hline w1123 & $2.85(5.25)$ & $-1.91(\mathbf{0 . 4 9})$ & $2.77(5.16)$ & $-1.43(\mathbf{0 . 9 6})$ \\
\hline Expr. & \multicolumn{2}{|c|}{$4.76[33-35]$} & \multicolumn{2}{|l|}{$4.20[36-38]$} \\
\hline
\end{tabular}

\subsection{Acidity and Solvent Dependence}

Since the service environment in many chemical processes is made up of a variety of mainly non-polar molecules rather than water, the acidity calculations have been performed for various solvents, spaning a full range of dielectric constants. As the dissociation process creates two ions from neutral species, it is expected to be strongly 
influenced by the dielectric properties or polarity of the surrounding medium and the equilibrium under these conditions would lie considerably closer to the left than in aqueous solution with the water molecules in the dissociation process occurring in stoichiometric amounts rather than as a solvent.

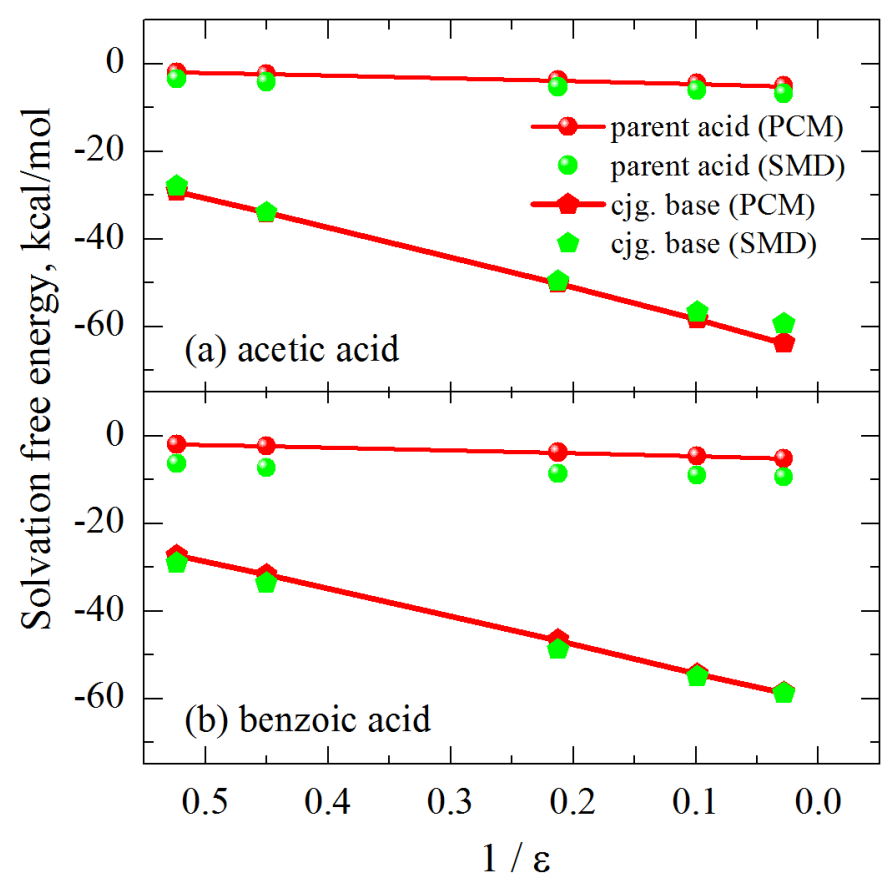

Figure 4. Solvation free energies of un-hydrated parent acids and their respective anions as a function of solvent's dielectric constant

Figure 4 displays the variation of solvation free energies as a function of solvent for undissociated acids and their respective conjugate bases. The solvation free energy of the ionic conjugate base is much sensitive to solvent variation than that of the neutral parent acid. According to Born equation [39,40], dielectric constant and ion radius are primary factors that contribute to electrostatic interaction. In case of ionic species, the electrostatic term is more dominant compared to the non-electrostatic one. Therefore the effect induced by solvent selection on $\Delta \mathrm{G}_{\text {solv }}$ as seen in Figure $\mathbf{4}$ comes from the change in electrostatic interactions between ions. Born equation denotes that the electrostatic energy is inversely proportional to solvent's dielectric constant and that could be implied from Figure 4. 
It might be expected that any water molecules present in non-polar solvents would tend to cluster around the acid species. Figure $\mathbf{5}$ presents the $\mathrm{pK}_{\mathrm{a}}$ values calculated for several cluster models that reproduce fair experimental data (in aqueous solution) as shown in previous section. The same thermodynamic cycle and dissociation constant equation as used for the aqueous solvent were used, except that the standard state of water was corrected to $1.0 \mathrm{M}$, instead of $55.4 \mathrm{M}$ and the calculated $\Delta G_{\text {solv }}^{0}\left(\mathrm{H}_{3} \mathrm{O}^{+}\right)$in organic solvents is used instead of the empirical values. The theoretical calculations for organic solvents without corrected $\Delta G_{\text {solv }}^{0}\left(\mathrm{H}_{3} \mathrm{O}^{+}\right)$result in relatively high values of $\mathrm{pK}_{\mathrm{a}}$, compared to experimental measurements. For instance, the calculated $\mathrm{pKa}$ for acetic acid in acetone, using the w0 model, is 37.66 , which is much higher than the experimental report of 18.91 using the potentiometric titration method [41]. However, if a value of $110.2 \mathrm{kcal} / \mathrm{mol}$ is roughly used for $\Delta G_{\text {solv }}^{0}\left(\mathrm{H}_{3} \mathrm{O}^{+}\right)$in acetone, the resulted $\mathrm{pKa}$ would be significantly reduced (to 12.73).

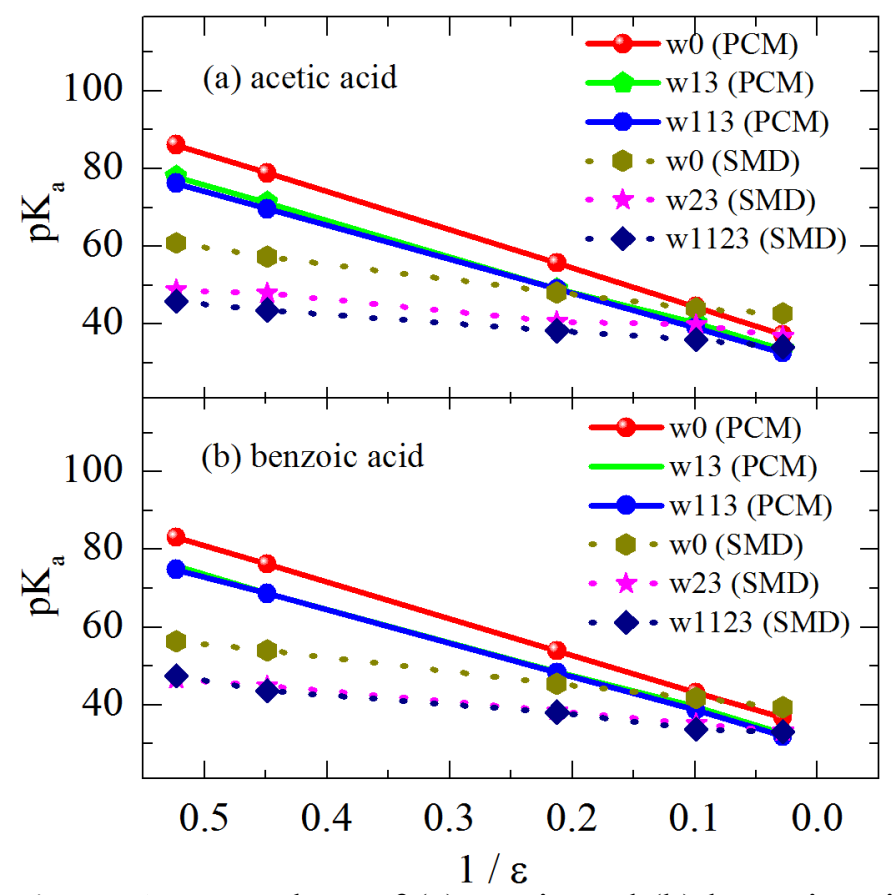

Figure 5. pKa values of (a) acetic and (b) benzoic acids as a function of solvent's dielectric constant, using equation (4) and thermodynamic cycle in Figure 2

Replacing the acid by water in equation (4) and assuming the ion-product constant of water $\mathrm{K}_{\mathrm{w}}=\left[\mathrm{H}_{3} \mathrm{O}^{+}\right]\left[\mathrm{OH}^{-}\right]=10^{-14}$ and $\left[\mathrm{H}_{2} \mathrm{O}\right]=55.4 \mathrm{M}$ at $25^{\circ} \mathrm{C}$, the $\mathrm{pK}_{\mathrm{a}}$ of water can be 
predicted as 15.74. In organic solvents as shown in Figure 5, the $\mathrm{pK}_{\mathrm{a}}$ values of acetic acid and benzoic acid could be as high as 80 (using PCM model) and 60 (using SMD model) in low dielectric environment such as heptane. This could raise a question about the validity of the dissociation model, whether the water could still act as a proton acceptor with regard to these acids. However, the rule of higher acidity constant in lower dielectric environment found above should also be applied here towards water. When water is presented in the organic solvent, its ion-product constant and standard concentration change and subsequently make it less "acidic". Therefore, in any environment, the presented thermodynamic cycle is still valid with water acting as a base in the acid-base reaction.

It is indicated that the tendency of losing a proton is higher in strong dielectric solvent. The solvent dependence obtained from SMD is less than that from PCM model, but the the $p K_{a}$ values in both cases could also be described as a linear function of $\varepsilon^{-1}$.

\subsection{Non-aqueous proton acceptors}

Through the course of attempting to find minimum energy geometries for proton solvation in non-aqueous media, it was found that there are two primary patterns via which protons may be "solvated" by organic molecules. These correspond to the reaction pathways described in equations (7) and (9) above. We style these pathways I and II. Pathway I involves a proton addition to an unsaturated bond, leading to the formation of a carbocation molecule. This pathway was observed for acetone and benzene solvents (Figure 6). Pathway II, on the other hand, involves an elimination reaction. The proton adds to a basic portion of the molecule and eliminates the protonated base via R-B bond scission. The remaining portion of the molecule is also a carbocation. This pathway was observed for straight-chain alkanes (including n-heptane), carbon tetrachloride, chloroform and dichloroethane (Figure 6). These solvents were selected as they span a range of dielectric constants.

Table 3. Gibbs free energy of acid-base reaction $\left(\Delta \mathrm{G}_{\text {solution }}, \mathrm{kcal} / \mathrm{mol}\right)$ with aqueous $\left(\mathrm{H}_{2} \mathrm{O}\right.$ molecule) and non-aqueous proton acceptors (organic solvent molecule)

\begin{tabular}{|l|l|l|l|}
\hline Solvents & Dielectric & Acetic acid & Benzoic acid \\
\hline
\end{tabular}




\begin{tabular}{|l|l|l|l|l|l|}
\hline & constant, $\varepsilon$ & $\begin{array}{l}\text { Water as } \\
\text { proton } \\
\text { acceptor }\end{array}$ & $\begin{array}{l}\text { Non-aqueous } \\
\text { proton acceptor } \\
\text { (organic solvent) }\end{array}$ & $\begin{array}{l}\text { Water as } \\
\text { proton } \\
\text { acceptor }\end{array}$ & $\begin{array}{l}\text { Non-aqueous } \\
\text { proton acceptor } \\
\text { (organic solvent) }\end{array}$ \\
\hline n-heptane & 1.91 & 117.42 & 132.02 & 113.30 & 127.90 \\
\hline carbontetrachloride & 2.22 & 107.53 & 103.23 & 103.94 & 99.63 \\
\hline benzene & 2.27 & 106.41 & 99.51 & 102.87 & 95.97 \\
\hline chloroform & 4.71 & 75.94 & 84.97 & 73.61 & 82.64 \\
\hline dichloroethane & 10.12 & 60.62 & 76.11 & 58.76 & 74.25 \\
\hline acetone & 20.49 & 53.75 & 41.11 & 52.93 & 40.29 \\
\hline
\end{tabular}

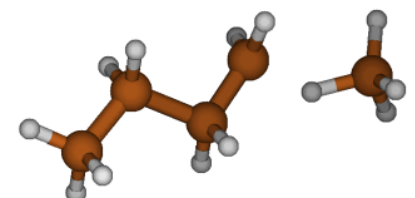

(a)

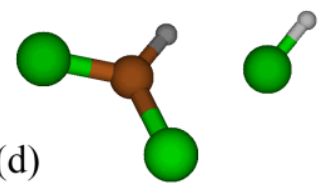

(b)

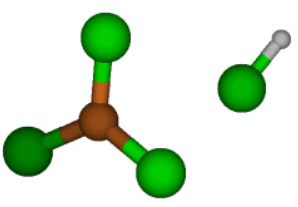

(c)

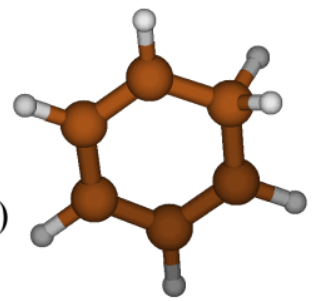

(e)

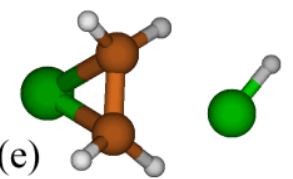

(f)

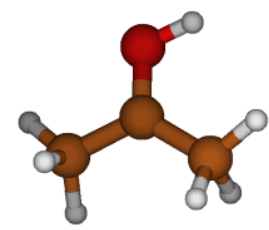

Figure 6. Optimized structures of non-aqueous proton acceptors, performed using the B3LYP/PCM/6-31+G(d,p) method: a) straight-chain alkanes (pathway II, pentane is presented), b) carbontetrachloride (pathway II), c) benzene (pathway I), d) chloroform (pathway II), e) dichloroethane (pathway II) and f) acetone (pathway I) 


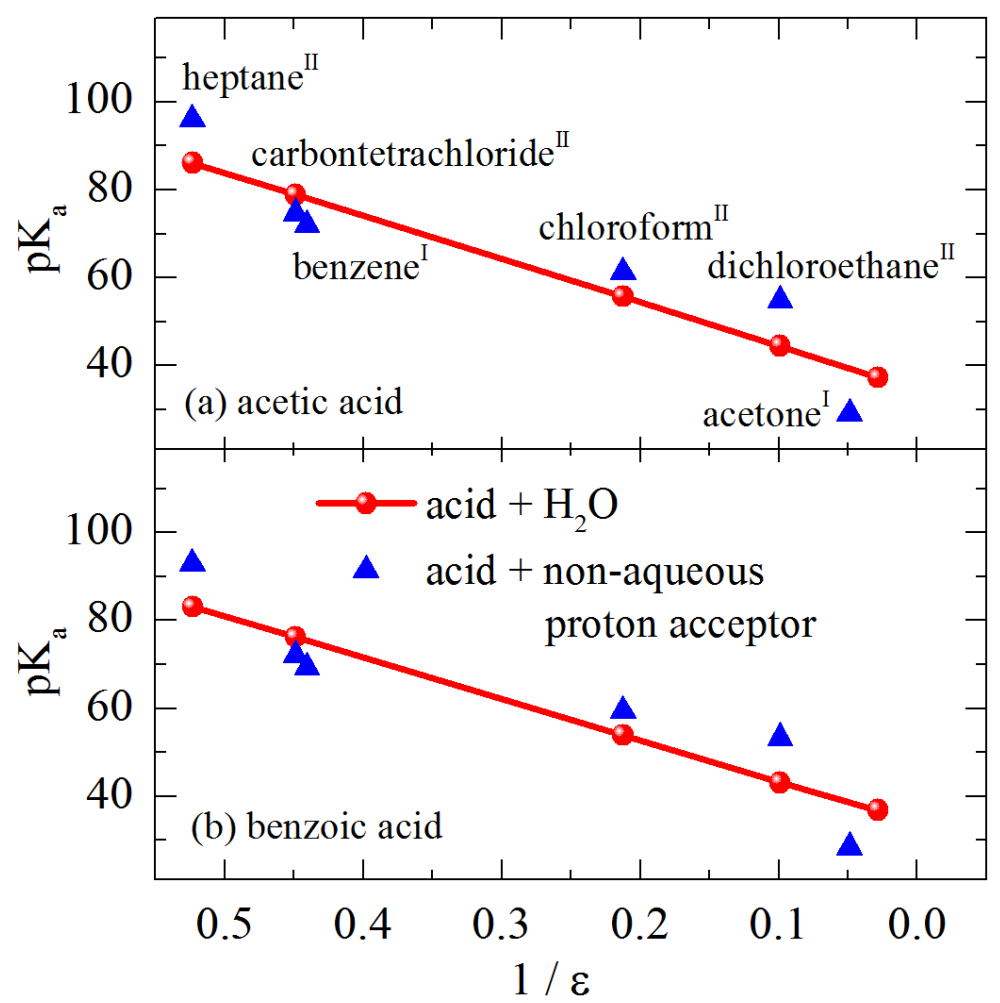

Figure 7. pKa values of acetic (a) and benzoic acids (b) as a function of solvent's dielectric constant, using equation (7) and (9) and thermodynamic cycles in Figure 3. The superscipt indicates the pathway observed for the correspoding solvent while involving in the dissociation reaction with acids

The same procedure was used to determine $\Delta \mathrm{G}_{\text {solution }}$ for these alternative acidbase reactions, and, subsequently, the $\mathrm{pK}_{\mathrm{a}}$. The free energies for these reactions at $298 \mathrm{~K}$ are shown in Table 3, along with the values where water is assumed to be the proton acceptor. The calculated $\Delta \mathrm{G}_{\text {solution }}$ values indicate that the dissociation reaction is endothermic in those selected solvents, regardless of the thermodynamic cycles and proton aceptors that used (aqueous and non-aqueous). However, the energies provided for the reaction to occur would be less in higher dilectric environment.

The $\mathrm{pK}_{\mathrm{a}}$ results for both cases are shown in Figure 7. We observe from this figure that the $\mathrm{pK}_{\mathrm{a}}$ values under this assumption (when no trace water is present and the organic solvent acts as the proton acceptor) are very similar to those values obtained when we assume that water is present. Furthermore, the general trend of decreasing $\mathrm{pK}_{\mathrm{a}}$ with increasing dielectric constant is preserved, which implies that acidity has as much to do 
with dielectric stabilization of the ionic products as it does with the chemistry of proton donation to the surrounding medium.

\section{Conclusion}

It was confirmed that using cluster models with water help improve the accuracy of $\mathrm{pK}_{\mathrm{a}}$ calculations. In general, the incorporation of acid molecule with more water would result in higher dissociation constant values from theoretical calculations. The water molecule can be introduced to different clustering position and the higher acidity can be observed in order: position 1 < position 2 < position 3 . However, the choice on cluster models might be subject to the selection of solvent model and the theoretical level of quantum chemistry calculations. For instance, using B3LYP/PCM, supermolecules with hydration numbers of two (w13) or three (w112, w113, w123) reproduce accurately the experimental values of acidity constants while a hydration number of two (w23) or four (w1123) would be recommended in case of M06-2X/SMD.

The effect of surrounding environment is more pronounced on the solvation of ionic species than on that of neutral acids. Dielectric constant is the primary factor that determines the solvent efffect. When water is present and assumed to be the proton acceptor, acid dissociation constants $\left(\mathrm{pK}_{\mathrm{a}}\right)$ exibit a linear variation of $\varepsilon^{-1}$. When water is absent and the organic solvent molecule participate directly in the dissociation reaction, the calculated $\mathrm{pK}_{\mathrm{a}}$ are similar, compared to the previous case (with water acting as the proton acceptor). In addition, $\mathrm{pK}_{\mathrm{a}}$ values change in similar manners with respect to continuum dielectric constant, in both cases of aqueous and alternative non-aqueous proton acceptors.

\section{Notes}

The authors declare no competing financial interests.

Acknowledgements. The authors acknowledge the use of high performance computing resources through the Institutional Computing Program at LANL for the completion of this research. The Los Alamos National Laboratory is operated by Los Alamos National 
Security LLC for the National Nuclear Security Administration of the U.S. Department of Energy under contract DE-AC52-06NA25396. 


\section{REFERENCES}

[1] G.A.A. Saracino, R. Improta, V. Barone, Chem Phys Lett 373 (2003) 411.

[2] C.O. da Silva, E.C. da Silva, M.A.C. Nascimento, J Phys Chem A 103 (1999) 11194.

[3] J.R. Fisher, H.L. Barnes, J Phys Chem-Us 76 (1972) 90.

[4] D.A. MacInnes, T. Shedlovsky, J Am Chem Soc 53 (1931) 2419.

[5] J.L. Oscarson, S.E. Gillespie, J.J. Christensen, R.M. Izatt, P.R. Brown, J Solution Chem 17 (1988) 865.

[6] R.E. Mesmer, C.S. Patterson, R.H. Busey, H.F. Holmes, J Phys Chem-Us 93 (1989) 7483.

[7] K. Sue, T. Morita, K. Totsuka, Y. Takebayashi, S. Yoda, T. Furuya, T. Hiaki, J Chem Eng Data 55 (2010) 4823.

[8] K. Sue, F. Ouchi, K. Minami, K. Arai, J Chem Eng Data 49 (2004) 1359.

[9] K. Sue, T. Usami, K. Arai, J Chem Eng Data 48 (2003) 1081.

[10] T. Xiang, K.P. Johnston, J Solution Chem 26 (1997) 13.

[11] Y.H. Jang, S. Hwang, S.B. Chang, J. Ku, D.S. Chung, J Phys Chem A 113 (2009) 13036.

[12] K.R. Adam, J Phys Chem A 106 (2002) 11963.

[13] J.R. Pliego, Chem Phys Lett 367 (2003) 145.

[14] D.M. Du, M. Qin, Z.Y. Zhou, A.P. Fu, Int J Quantum Chem 112 (2012) 351.

[15] W.H. Richardson, C. Peng, D. Bashford, L. Noodleman, D.A. Case, Int J Quantum Chem 61 (1997) 207.

[16] I.A. Topol, G.J. Tawa, S.K. Burt, A.A. Rashin, J Phys Chem A 101 (1997) 10075.

[17] Y.H. Jang, L.C. Sowers, T. Cagin, W.A. Goddard, J Phys Chem A 105 (2001) 274.

[18] C.P. Kelly, C.J. Cramer, D.G. Truhlar, J Phys Chem A 110 (2006) 2493.

[19] J.R. Pliego, J.M. Riveros, J Phys Chem A 106 (2002) 7434.

[20] M.D. Liptak, G.C. Shields, Int J Quantum Chem 85 (2001) 727.

[21] H.H. Pham, C.D. Taylor, N.J. Henson, J Phys Chem B 117 (2013) 868.

[22] J.M. Ho, M.L. Coote, J Chem Theory Comput 5 (2009) 295.

[23] J.M. Ho, M.L. Coote, Theor Chem Acc 125 (2010) 3. 
[24] J.M. Ho, M.L. Coote, Wires Comput Mol Sci 1 (2011) 649.

[25] F.Z. Ding, J.M. Smith, H.B. Wang, J Org Chem 74 (2009) 2679.

[26] B. Ghalami-Choobar, A. Ghiami-Shomami, Comput Theor Chem 1018 (2013) 66.

[27] L.M. Mihichuk, G.W. Driver, K.E. Johnson, Chemphyschem 12 (2011) 1622.

[28] H.Z. Yu, Y.M. Yang, L. Zhang, Z.M. Dang, G.H. Hu, J Phys Chem A 118 (2014) 606.

[29] S. Miertus, E. Scrocco, J. Tomasi, Chem Phys 55 (1981) 117.

[30] M.J. Frisch, G.W. Trucks, H.B. Schlegel, G.E. Scuseria, M.A. Robb, J.R. Cheeseman, G. Scalmani, V. Barone, B. Mennucci, G.A. Petersson, e. al., Gaussian 09, Revision A.1. Gaussian, Inc., Wallingford CT, 2009.

[31] C. Colominas, J. Teixido, J. Cemeli, F.J. Luque, M. Orozco, J Phys Chem B 102 (1998) 2269.

[32] A.V. Marenich, C.J. Cramer, D.G. Truhlar, J Phys Chem B 113 (2009) 6378.

[33] H.S. Harned, R.W. Ehlers, J Am Chem Soc 55 (1933) 652.

[34] A. Kumar, Thermochim Acta 439 (2005) 154.

[35] D.A. Lown, H.R. Thirsk, J Chem Soc Farad T 168 (1972) 1982.

[36] A. Habibi-Yangjeh, M. Danandeh-Jenagharad, M. Nooshyar, B Kor Chem Soc 26 (2005) 2007.

[37] A.V. Jones, H.N. Parton, T Faraday Soc 48 (1952) 8.

[38] L.E. Strong, T. Kinney, P. Fischer, J Solution Chem 8 (1979) 329.

[39] P.W. Atkins, A.J. Macdermott, J Chem Educ 59 (1982) 359.

[40] H. Bartnicka, I. Bojanowska, M.K. Kalinowski, Aust J Chem 44 (1991) 1077.

[41] M. Czaja, A. Kozak, M. Makowski, L. Chmurzynski, J Chem Thermodyn 37 (2005) 783. 